\title{
DÜZENSİZ, SABİT VEYA ÖTELENEN/DÖNEN DÜZLEMLERDE ISI İLETİMİ İÇİN SAYISAL FORMÜLASYON GELİŞTÍRİLMESİ
}

\author{
Saad bin Mansoor*, Abuzer Ozsunar **, Bekir Sami Yilbas* \\ * King Fahd University of Petroleum and Minerals, Mechanical Engineering Department, Dhahran, Saudi \\ Arabia \\ ** Gazi Üniversitesi, Mühendislik Fakültesi, Makina Mühendisliği Bölümü, 06570 Maltepe/Ankara, Türkiye \\ $\underline{\text { saadbin@kfupm.edu.sa, ozsunar@gazi.edu.tr, bsyilbas@kfupm.edu.sa }}$
}

(Geliş/Received: 10.02.2014; Kabul/Accepted: 06.11.2014)

ÖZET

Riemannian uzayı gibi, iki üç boyutlu bütün Euclidean koordinat sistemlerine uygulanabilecek, 1Sı iletim denkleminin çözümü için yeni bir yaklaşım sunulmuştur. Denklem bileşenlerine ayrılmış olduğundan doğrudan sayısal hesaplamalarda kullanılabilir. Isı iletim denkleminin bu formu, düzensiz sabit veya ötelenen/dönen düzlemsel bölgelerde geçici sıcaklık alanının hesaplanması için bir yöntem oluşturulmasında kullanılır. Sadece sınır eğrileri ile tanımlanan düzensiz alanlarda düzlemsel ağlar oluşturmak için türetilen yöntem ayrıca tartışılmıştır.

Anahtar Kelimeler: Düzensiz, sabit, ötelenen/dönen düzlemlerde 1sı iletimi, HAD (CFD)

\section{A NUMERICAL FORMULATION OF HEAT CONDUCTION IN IRREGULAR, STATIC OR TRANSLATING/ROTATING, PLANAR DOMAINS}

\begin{abstract}
A new approach for the solution of heat conduction equation is presented, which is applicable to all coordinate systems in two- and three-dimensional Euclidean as well as Riemannian space. The equation is in component form and is directly applicable to the numerical computation This general form of the heat conduction equation is then used to construct a technique for calculating the transient temperature field in irregular, static or translating/rotating, planar domains. A method to generate planar grids in irregular domains, defined only by their boundary curves, is also discussed.
\end{abstract}

Keywords: Heat Conduction in irregular, translating/rotating planar domains, CFD.

\section{GÍRIŞ (INTRODUCTION)}

Gerçek hayatta 1sı iletimi problemleri kare, daire, eliptik vs. gibi standart geometrilerden farklı düzensiz domainlerden oluşur. $\mathrm{Bu}$ yüzden, problemi sonlu farklar yönetmiyle çözerken, eğri sınırlar birçok küçük düz çizgilere bölünür. Hem doğruluk hem de çözüm yönteminin verimi azalır. Ayrıca, sınır şartlarını uygulamada zorluklar çıkar.

$\mathrm{Bu}$ uygulama zorluğundan kaçınmanın bir yolu, sayısal ağ üretimi tekniği (Numerical Grid Generation-NGG) yoluyla body-fitted coordinate kullanmaktır [1]. Bu tekniğin arkasındaki temel fikir, düzensiz bölgeyi düzenli bölgeye dönüştürdükten sonra dönüştürülmüş 1S1 iletimi denklemini bu bölgede çözmektir. Temel denklemlerin dönüşümü yorucu ve oldukça zor bir iştir. Ancak, tansör matematiği kullanılarak bu iş kolaylıkla ve sistematik bir yöntemle aşılabilir. Bu makalede, konuyu çok genel bir şekilde ele almayı sağlamak için NGG tekniği tansör matematiğiyle birleştirilmiştir.

Mühendislik problemlerinin sayısal çözümünde, hareketli ağ yöntemi kabul edilmiş birçok çalışmada uygulanmıştır. Zamana bağlı kısmi diferansiyel 
denklemlerin sayısal çözümü için, uyarlanabilir hareketli ağ tekniği (The adaptive moving grid techniques) Wathen [2] tarafindan önerilmiştir. En küçük kareler normunda kısmi diferansiyel denklemi çözümünün, parçalı doğrusal sonlu elemanlar yaklaşımıyla, optimal hareketli ağların sayısal çözümünü açıklamıştır. McCorquodale ve arkadaşları tarafından [3], düzensiz çözüm alanlarında irregular domainlerde 1sı denklemi için, kartezyen ağ gömülü sınır metodu araştırılmıştır. McCorquodale vd. [3] hareketli sinır durumunda, Crank-Nicolson zaman ayrıklaştırmanın kullanımının tutarsız olduğunu ve bunun yerine, $\mathrm{L}_{\mathrm{o}}$-kararlı kapalı Runge-Kutta yöntemi kulanmanın gerekli olduğunu göstermişlerdir. Beckett vd. [4] iki boyutlu Stefan problemlerinin çözümü için hareketli kafes sonlu elemanlar yöntemini incelemişlerdir. Yarı kapalı Galerkin sonlu elemanlar yöntemini doğrusal temel fonksiyonlar kullanarak entalpi denklemini ayrıklaştırmışlardır. Ön yüzü çıkıntılı ve arayüzeyi değişken bir topolojiye sahip problemler için hareketli sonlu elemanlar yöntemini uygulamışlardır. Kuvyrkin ve Titov [5], hareketli sınırlı 1S1 iletimini çalışmışlardır. Çakışmayan koordinatların işlerliğinin geçerliliğini ve ăg elemanlar üzerindeki ağırlık fonksiyonlarını sayısal bir örnekle göstermişlerdir. Gilmore ve Guceri [6], arayüzeyde Stefan şartlarında üç boyutlu iletim probleminin formülasyonu için uygulamışlardır. Hareketli arayüzeyi hesaplamak için sürekli güncellenen çözüm bölgesi üzerinde üç boyutlu kafes oluşturmak için eliptik tip ağ üretimi motodu kullandılar. Eriyen cisimlerde, iki boyutlu ve simetrik olmayan isıl tepkiyi belirlemek için Chin [7] tarafından sonlu elemanlar yöntemi geliştirilmiştir. Kısmi eriyen elemanların özelliklerini hesaplamak için genişletilmiş integral motodu formüle edilmiştir.

\section{Isı Transfer Analizi (Heat Transfer Analysis)}

Isı transfer analizinde, 1sı iletim denklemi için uygun tansör matematiği sunulmuş ve 1sitma problemine uygulaması gösterilmiştir. Sabit ve hareketli ağ sistemi için sayısal ağ üretimi yapılmıştır. Makalede, tansör analizinde kullanılan aralık ve toplama kuralları kullanılmıştır. Gösterimler şu şekilde yapılmıştır. , $\xi \rightarrow x^{1}, \eta \rightarrow x^{2}$ ve $x \rightarrow z^{1}, y \rightarrow z^{2}$ burada, $(\xi, \eta)$ eğrisel koordinatlar ve $(x, y)$ kartezyen koordinatlardır.

\subsection{Dönüşüm Bağıntıları (Transformation Relations)}

Şekil 1'de görüldüğü gibi her bir tarafı gelişigüzel sınırlanmış, kartezyen koordinat sisteminde düzensiz bölge olduğunu kabul edelim. Bu bölge, aşağıdaki dönüşümlerle $\xi-\eta$ koordinat sisteminde kare bölgeye dönüştürülebilir,

$$
\begin{array}{ll}
x=x(\xi, \eta), & 0 \leq \xi \leq 1 \\
y=y(\xi, \eta) & 0 \leq \eta \leq 1
\end{array}
$$

Örnek olarak,
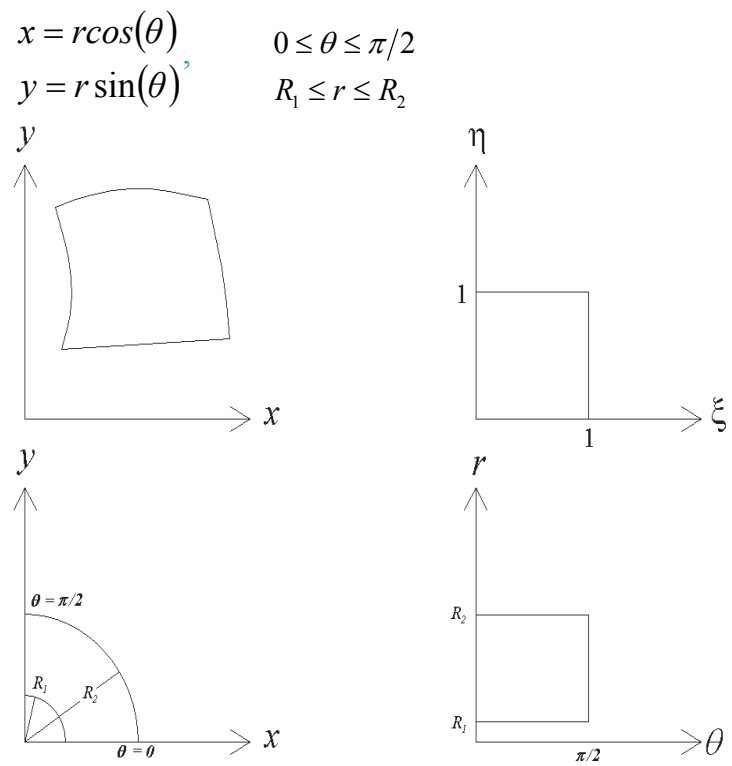

Şekil 1. Düzensiz bölgelerin dikdörtgen bölgelere dönüştürülmesi (Examples of irregular regions transforming in to regular regions).

Kutupsal koordinat sisteminde bir dikdörtgen, kartezyen koordinat sisteminde dairesel bir bölgeye dönüşür.

Denklem (1), sınırları eğri olan gelişigüzel bir bölge için genellikle bilinmez. Bununla birlikte, bir an bildiğimizi kabul edelim. Eğer çözüm alanı öteleniyor/dönüyor ise dönüşüm denklemleri aşağıdaki şekilde yazılabilir,

$$
\begin{array}{ll}
x=x(\xi, \eta, t) & 0 \leq \xi \leq 1 \\
y=y(\xi, \eta, t) & 0 \leq \eta \leq 1 \\
& t \geq 0
\end{array}
$$

$\mathrm{Bu}$ denklemler daima $\mathrm{x}-\mathrm{y}$ uzayında orjinal bölge, $\xi-\eta$ uzayında kare bölgeye dönüşecektir.

\subsection{Isı İletim Denklemine Uygulama (Application To Heat Conduction Equation)}

Herhangi bir koordinat sisteminde geçici rejimdeki 1s1 iletim denklemi Öklit (Euclidean) veya Reimann (Reimannian) uzayı ve herhangi bir boyutta bilinir,

$$
\frac{1}{\alpha} \frac{\partial T}{\partial t}=\nabla \cdot(\nabla T)
$$

Burada, T sicaklık, t zaman ve $\alpha$ isıl yayılım katsayısıdır. Isı yayılım katsayısı sabit olarak alınmıştır. Yukarıda verilen denklem sayısal 
hesaplamalar için kullanılamaz. Bu nedenle, denklem bileşen formda yazılmalıdır. Herhangi bir koordinat sisteminde, Laplace (Laplacian) $\nabla .(\nabla T)$ tansör matematiği kullanılarak belirlenir. Laplace operatorü, Laplace-Beltrami Operatorü olarak bilinir. Isı iletim denklemi şu şekilde verilir[8],

$$
\left.\frac{1}{\alpha} \frac{\partial T}{\partial t}\right|_{x^{i}}=g^{i j}\left(\frac{\partial^{2} T}{\partial x^{i} x^{j}}-\left\{\begin{array}{c}
k \\
i j
\end{array}\right\} \frac{\partial T}{\partial x^{k}}\right)
$$

Yukarıda verilen denklemdeki semboller: $g_{i j}$ çözüm alanında herhangi bir noktada metrik tansördür (kovaryant metrik tansör) ve şöyle tanımlanır[9],

$$
\left[g_{i j}\right]=\left[\begin{array}{ll}
\left(\frac{\partial x}{\partial \xi}\right)^{2}+\left(\frac{\partial y}{\partial \xi}\right)^{2} & \frac{\partial x}{\partial \xi} \frac{\partial x}{\partial \eta}+\frac{\partial y}{\partial \xi} \frac{\partial y}{\partial \eta} \\
\frac{\partial x}{\partial \xi} \frac{\partial x}{\partial \eta}+\frac{\partial y}{\partial \xi} \frac{\partial y}{\partial \eta} & \left(\frac{\partial x}{\partial \eta}\right)^{2}+\left(\frac{\partial y}{\partial \eta}\right)^{2}
\end{array}\right]
$$

Buradaki kısmi türevler, denklem (1) tarafından hesaplanır. Birleşik metrik tansör (kontvaryant metrik tansör) şu şekilde tanımlanır,

$\left[g^{i j}\right]=\left[g_{i j}\right]^{-1}=\frac{1}{J^{2}}\left[\begin{array}{cc}\left(\frac{\partial x}{\partial \eta}\right)^{2}+\left(\frac{\partial y}{\partial \eta}\right)^{2} & -\left(\frac{\partial x}{\partial \xi} \frac{\partial x}{\partial \eta}+\frac{\partial y}{\partial \xi} \frac{\partial y}{\partial \eta}\right) \\ -\left(\frac{\partial x}{\partial \xi} \frac{\partial x}{\partial \eta}+\frac{\partial y}{\partial \xi} \frac{\partial y}{\partial \eta}\right) & \left(\frac{\partial x}{\partial \xi}\right)^{2}+\left(\frac{\partial y}{\partial \xi}\right)^{2}\end{array}\right]$

burada $J=\sqrt{\left|g_{i j}\right|}=\frac{\partial x}{\partial \xi} \frac{\partial y}{\partial \eta}-\frac{\partial y}{\partial \xi} \frac{\partial x}{\partial \eta}$ Jacobian'dır.

$\left\{\begin{array}{c}k \\ i j\end{array}\right\}$ Băg katsayıları olarak bilinir ve şu şekilde hesaplanır[10]:

$\left\{\begin{array}{c}k \\ i j\end{array}\right\}=\frac{1}{2} g^{k m}\left(\frac{\partial g_{i m}}{\partial x^{j}}+\frac{\partial g_{m j}}{\partial x^{i}}-\frac{\partial g_{i j}}{\partial x^{m}}\right)$

$\xi-\eta$ koordinatları cinsinden denklem (3) şu şekilde yazılır,

$\left.\frac{1}{\alpha} \frac{\partial T}{\partial t}\right|_{\xi, \eta}=g^{11} \frac{\partial^{2} T}{\partial \xi^{2}}+2 g^{12} \frac{\partial^{2} T}{\partial \xi \partial \eta}+g^{22} \frac{\partial^{2} T}{\partial \eta^{2}}$

$\left.-\left(g^{11}\left\{\begin{array}{c}1 \\ 11\end{array}\right\}+2 g^{12}\left\{\begin{array}{c}1 \\ 12\end{array}\right\}+g^{22}\left\{\begin{array}{c}1 \\ 22\end{array}\right\}\right)\right) \frac{\partial T}{\partial \xi}-\left(g^{11}\left\{\begin{array}{c}2 \\ 11\end{array}\right\}+2 g^{12}\left\{\begin{array}{c}2 \\ 12\end{array}\right\}+g^{22}\left\{\begin{array}{c}2 \\ 22\end{array}\right\}\right) \frac{\partial T}{\partial \eta}$

Eğer fiziksel çözüm alanı öteleniyor/dönüyor ise, yukarıdaki ısı iletim denklemi biraz değiştirilecektir. Genellikle eğrisel koordinatlarda zamanın fonksiyonu olduğu için 1sı iletim denklemi (3\&4) ün sol tarafi yazılırsa,

$\frac{1}{\alpha}\left(\left.\frac{\partial T}{\partial t}\right|_{x^{1}, x^{2}}-\frac{\partial T}{\partial z^{i}} \frac{\partial z^{i}}{\partial t}\right)$

Burada $z^{i}$ kartezyen koordinatlardır. $\mathrm{Bu}$ ifade denklem (3)'te yerine yazılarak yeniden düzenlenirse,

$\left.\frac{1}{\alpha} \frac{\partial T}{\partial t}\right|_{x^{1}, x^{2}}=g^{i j}\left(\frac{\partial^{2} T}{\partial x^{i} \partial x^{j}}-\left\{\begin{array}{c}k \\ i j\end{array}\right\} \frac{\partial T}{\partial x^{k}}\right)+\frac{1}{\alpha} \frac{\partial T}{\partial z^{i}} \frac{\partial z^{i}}{\partial t}$

$\xi-\eta$ koordinat cinsinden, denklem (6) şu şekilde yazılır,

$\left.\frac{1}{\alpha} \frac{\partial T}{\partial t}\right|_{\xi, \eta}=g^{11} \frac{\partial^{2} T}{\partial \xi^{2}}+2 g^{12} \frac{\partial^{2} T}{\partial \xi \partial \eta}+g^{22} \frac{\partial^{2} T}{\partial \eta^{2}}-\left(g^{11}\left\{\begin{array}{c}1 \\ 11\end{array}\right\}+2 g^{12}\left\{\begin{array}{c}1 \\ 12\end{array}\right\}+g^{22}\left\{\begin{array}{c}1 \\ 22\end{array}\right\}\right) \frac{\partial T}{\partial \xi}$

$-\left(g^{11}\left\{\begin{array}{c}2 \\ 11\end{array}\right\}+2 g^{12}\left\{\begin{array}{c}2 \\ 12\end{array}\right\}+g^{22}\left\{\begin{array}{c}2 \\ 22\end{array}\right\}\right) \frac{\partial T}{\partial \eta}+\frac{1}{\alpha}\left(\frac{\partial T}{\partial x} \frac{\partial x}{\partial t}+\frac{\partial T}{\partial y} \frac{\partial y}{\partial t}\right)$

Fakat [12],

$\frac{\partial T}{\partial x}=\frac{\partial T}{\partial \xi} \frac{\partial \xi}{\partial x}+\frac{\partial T}{\partial \eta} \frac{\partial \eta}{\partial x}=\frac{1}{J}\left(\frac{\partial T}{\partial \xi} \frac{\partial y}{\partial \eta}-\frac{\partial T}{\partial \eta} \frac{\partial y}{\partial \xi}\right)$

$\frac{\partial T}{\partial y}=\frac{\partial T}{\partial \xi} \frac{\partial \xi}{\partial y}+\frac{\partial T}{\partial \eta} \frac{\partial \eta}{\partial y}=\frac{1}{J}\left(-\frac{\partial T}{\partial \xi} \frac{\partial x}{\partial \eta}+\frac{\partial T}{\partial \eta} \frac{\partial x}{\partial \xi}\right)$

Son olarak Denklem (6) şöyle yazılır,

$$
\begin{aligned}
\left.\frac{1}{\alpha} \frac{\partial T}{\partial t}\right|_{\xi, \eta}= & g^{11} \frac{\partial^{2} T}{\partial \xi^{2}}+2 g^{12} \frac{\partial^{2} T}{\partial \xi \partial \eta}+g^{22} \frac{\partial^{2} T}{\partial \eta^{2}} \\
& -\left(g^{11}\left\{\begin{array}{c}
1 \\
11
\end{array}\right\}+2 g^{12}\left\{\begin{array}{c}
1 \\
12
\end{array}\right\}+g^{22}\left\{\begin{array}{c}
1 \\
22
\end{array}\right\}+\frac{1}{\alpha J}\left(\frac{\partial y}{\partial t} \frac{\partial x}{\partial \eta}-\frac{\partial x}{\partial t} \frac{\partial y}{\partial \eta}\right)\right) \frac{\partial T}{\partial \xi} \\
& -\left(g^{11}\left\{\begin{array}{c}
2 \\
11
\end{array}\right\}+2 g^{12}\left\{\begin{array}{c}
2 \\
12
\end{array}\right\}+g^{22}\left\{\begin{array}{c}
2 \\
22
\end{array}\right\}+\frac{1}{\alpha J}\left(\frac{\partial x}{\partial t} \frac{\partial y}{\partial \xi}-\frac{\partial y}{\partial t} \frac{\partial x}{\partial \xi}\right)\right) \frac{\partial T}{\partial \eta}
\end{aligned}
$$

Denklem (6) ve (7), $\xi-\eta$ koordinatlarında sirasıyla durağan ve ötelenen/dönen alanlarda 1 sı iletim denkleminin son şeklidir.

Yukarıdaki 1sı iletim denklemleri tüm koordinat sistemlerinde geçerlidir. Ayrıca, Euclidean uzayının bir alt kümesi olan Riemannian uzayında da geçerlidir. Bunun anlamı, yukarıda verilen denklemler, kabuğun gömülü olduğu üç boyutlu Euclidean uzayı referans verilmeksizin sadece yüzey üzerinde tanımlanmış koordinatlar cinsinden küresel kabuğun yüzeyi üzerinde 1 sı iletim denklemi kullanılabilir. $\mathrm{Bu}$ durumda dönüşüm denklemleri, sırasıyla, durağan ve ötelenen/dönen çözüm alanları için şu şekilde yazılır, 


$$
\begin{array}{llll}
x=x(\xi, \eta) & & x=x(\xi, \eta, t) & 0 \leq \xi \leq 1 \\
y=y(\xi, \eta) & 0 \leq \xi \leq 1 & y=y(\xi, \eta, t) & 0 \leq \eta \leq 1 \\
z=y(\xi, \eta) & 0 \leq \eta \leq 1 & z=y(\xi, \eta, t) & t \geq 0
\end{array}
$$

Denklem (7)'deki $\{\partial x / \partial t, \partial y / \partial t\}$ türevleri, ağ hızları olarak bilinir. Ağ durağansa öteleme/dönme yoksa bu türevler sıfır olur. Bunun yanında, ağ öteleniyor/dönüyorsa türevler sıfır değildir ve Denklem (2)'den hesaplanır. Dönüşüm denklemleri olarak bilinen bu yaklaşımla gelişigüzel deforme olan yüzey için isı iletim denkleminin türevi gerçekleştirilir.

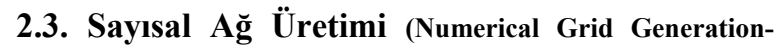 NGG)}

Daha önce bahsedildiği gibi, sadece bilinen eğri sınırlar ile tanımlanmış herhangi bir düzlem bölge için dönüşüm denklemleri (1 \& 2) genel bilinmeyenlerdir. Analitik çözümler için, bu bağıntılar konformal dönüşüm teorisinden bulunabilir. Bununla birlikte, sayısal hesaplamalar için, dönüşüm denklemlerini (1 \& 2)'nin fonksiyon formunu bimemize gerek yoktur. $\mathrm{Bu}$ denklemlerin tabular formunu bilmek yeterlidir. Tabular form, $(\xi-\eta)$ koordinatları sabit değerleri için, $(x-y)$ koordinatlarının sayısal değerlerini verir. $\mathrm{Bu}$ değerler bilgisayar hafizasında diziler olarak depolanır ve bu, dönüşüm denklemlerimizi oluşturur. $\mathrm{Bu}$ dizilerden, gerekli türevler, metrik tansörler, ilişki katsayıları (the connexion coefficients) ve ağ hızları standart sonlu farklar formülleri yoluyla belirlenebilir. $\mathrm{Bu}$ tabular formları bulmak için, durağan çözüm alanlarının dönüşüm denklemlerinin çözümlerinin Laplace denklemleri olduğunu kabul edebiliriz[11]. NGG tekniğinin kulanımı için anahtar fikrin birisi budur ve referans[1]'de tartışılmıştır. Aşağıda verilen eşitlikler durağan ağların üretilmesi için kullanılabilir,

$$
\begin{array}{ll}
\alpha \frac{\partial^{2} x}{\partial \xi^{2}}-2 \beta \frac{\partial^{2} x}{\partial \xi \partial \eta}+\gamma \frac{\partial^{2} x}{\partial \eta^{2}}=0 & 0 \leq \xi \leq 1 \\
\alpha \frac{\partial^{2} y}{\partial \xi^{2}}-2 \beta \frac{\partial^{2} y}{\partial \xi \partial \eta}+\gamma \frac{\partial^{2} y}{\partial \eta^{2}}=0 & 0 \leq \eta \leq 1
\end{array}
$$

Burada,

$$
\alpha=\left(\frac{\partial x}{\partial \eta}\right)^{2}+\left(\frac{\partial y}{\partial \eta}\right)^{2}, \quad \beta=\frac{\partial x}{\partial \xi} \frac{\partial x}{\partial \eta}+\frac{\partial y}{\partial \xi} \frac{\partial y}{\partial \eta}, \quad \gamma=\left(\frac{\partial x}{\partial \xi}\right)^{2}+\left(\frac{\partial y}{\partial \xi}\right)^{2}
$$

Eğer ağ öteleniyor/dönüyor ise, dönüşüm denklemleri aşağıdaki denklemlerin çözümü yoluyla elde edilebilir.

$$
\begin{array}{cl}
\frac{1}{c_{x}} \frac{\partial x}{\partial t}=\frac{1}{J^{2}}\left(\alpha \frac{\partial^{2} x}{\partial \xi^{2}}-2 \beta \frac{\partial^{2} x}{\partial \xi \partial \eta}+\gamma \frac{\partial^{2} x}{\partial \eta^{2}}\right) & 0 \leq \xi \leq 1 \\
\frac{1}{c_{y}} \frac{\partial y}{\partial t}=\frac{1}{J^{2}}\left(\alpha \frac{\partial^{2} y}{\partial \xi^{2}}-2 \beta \frac{\partial^{2} y}{\partial \xi \partial \eta}+\gamma \frac{\partial^{2} y}{\partial \eta^{2}}\right) & t>0
\end{array}
$$

Burada $c_{x}$ ve $c_{y}$ sabittir ve birim mertebe değerlere sahiptir. Denklem (8 \& 9) irregular domainlerin sınırlarının bilinmesine göre, Dirichlet tipi sınır değer problemi veya başlangıç-sınır değer problemi olarak ele alınır. Bunlar sonlu farklar metodu yoluyla kolaylıkla çözülebilir. Bundan dolayı, yukarıdaki denklemler sayısal olarak çözülmesi sonucu, gerekli türevler, metrik tansörler, ilişki katsayıları ve ağ hızlarının belirlenmesiyle tabular formda dönüşüm denklemleri ( 1 \& 2) elde edilebilir. Bunlar elde edildikten sonra, geçici sıcaklık alanının sayısal çözümünü elde edebilmek için ısı iletim denklemi (5 \& 7)'de yerine konur.

2.4. Örnek Problem: Düzlemsel Eliptik Bir Plakada Kararlı Durumda Sıcaklık Dağılımı (Case Study: Steady-State Temperature Distribution In A Planar Elliptic Plate)

Şekil 2'de görüldü gibi düzlemsel metal bir plakayı dikkate alalım. Plakanın üst eliptik tarafı $100{ }^{\circ} \mathrm{C}$ ve kalan üç sınır $0{ }^{\circ} C^{\prime}$ dir. Problem eliptik metal plakanın kararlı durumda sıcaklık dağılımının bulunmasıdır. $\mathrm{Bu}$ problem regular sonlu farklar yöntemi ile çözülürse, Şekil 2'deki gibi bir ağ bulunur. Sayısal ağ üretim tekniği kullanıldığında ise, eliptik bölge $\xi-\eta$ uzayında bir kareye dönüştürülür. Bu Şekil 3'te görüldüğü gibi bir ağ dağılımı verir. Yönetici denklemler, ağ üretimi için sınır şartları ve ısı iletim denklemi aşağıda verilmiştir.

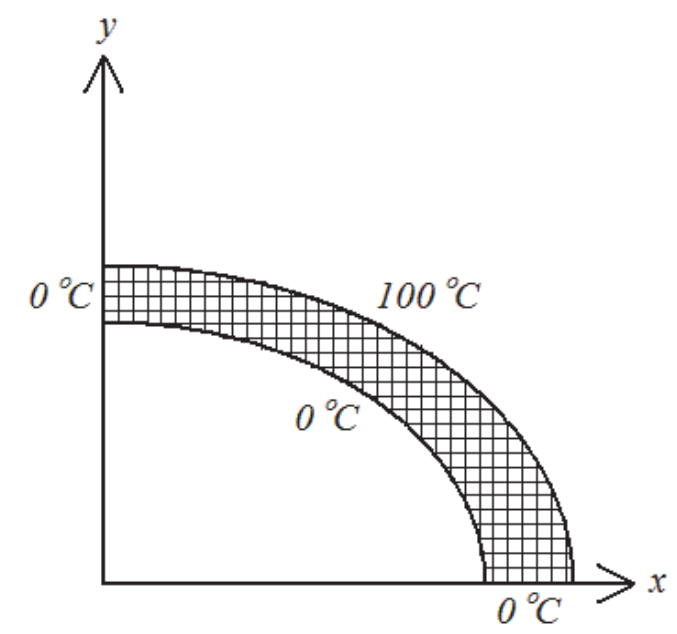

Şekil 2. Eliptik plaka üzerinde dikdörtgen 1zgara (Rectangular grid on an elliptic plate). 

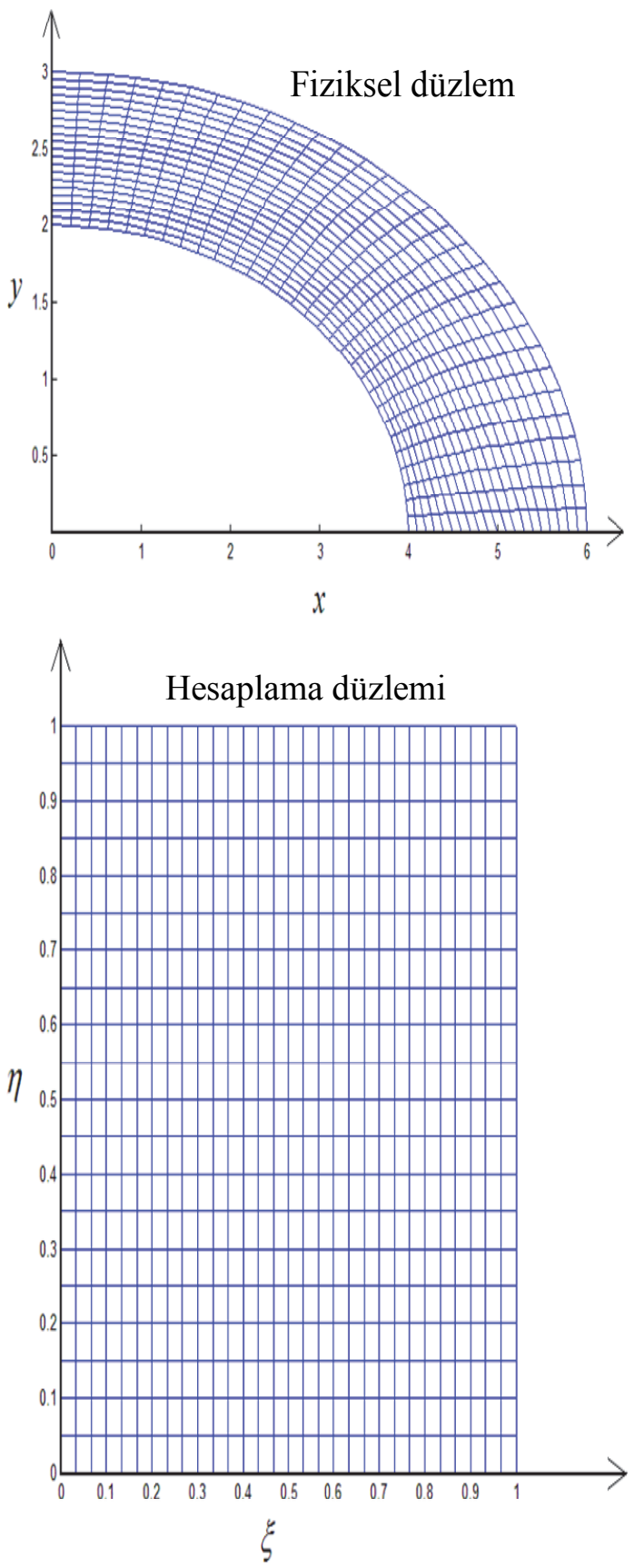

Şekil 3. $x-y$ uzayındaki fiziksel problemin ızgarasının $\xi-\eta \quad$ uzayında dikdörtgen 1zgara yapısına dönüştürülmesi (Rectangular grid in the $\xi-\eta$ space transforms in to a body-fitted grid in the $x-y$ space).

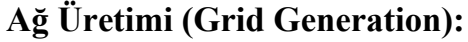

\section{Denklem (8),}

$$
\begin{array}{ll}
\alpha \frac{\partial^{2} x}{\partial \xi^{2}}-2 \beta \frac{\partial^{2} x}{\partial \xi \partial \eta}+\gamma \frac{\partial^{2} x}{\partial \eta^{2}}=0 & 0 \leq \xi \leq 1 \\
\alpha \frac{\partial^{2} y}{\partial \xi^{2}}-2 \beta \frac{\partial^{2} y}{\partial \xi \partial \eta}+\gamma \frac{\partial^{2} y}{\partial \eta^{2}}=0 & 0 \leq \eta \leq 1
\end{array}
$$

Burada,

$$
\alpha=\left(\frac{\partial x}{\partial \eta}\right)^{2}+\left(\frac{\partial y}{\partial \eta}\right)^{2}, \beta=\frac{\partial x}{\partial \xi} \frac{\partial x}{\partial \eta}+\frac{\partial y}{\partial \xi} \frac{\partial y}{\partial \eta}, \gamma=\left(\frac{\partial x}{\partial \xi}\right)^{2}+\left(\frac{\partial y}{\partial \xi}\right)^{2}
$$

Sınır Şartları:

$$
\begin{array}{ll}
x(0, \eta)=0 & y(0, \eta)=2+\eta \\
x(1, \eta)=4+2 \eta & y(1, \eta)=0 \\
x(\xi, 0)=4 \cos \frac{\pi(1-\xi)}{2} & y(\xi, 0)=2 \sin \frac{\pi(1-\xi)}{2} \\
x(\xi, 1)=6 \cos \frac{\pi(1-\xi)}{2} & y(\xi, 1)=3 \sin \frac{\pi(1-\xi)}{2}
\end{array}
$$

\section{Isı İletimi (Heat Conduction):}

\section{Denklem (5),}

$$
\begin{aligned}
\left.\frac{1}{\alpha} \frac{\partial T}{\partial t}\right|_{\xi, \eta \eta} & =g^{11} \frac{\partial^{2} T}{\partial \xi^{2}}+2 g^{12} \frac{\partial^{2} T}{\partial \xi \partial \eta}+g^{22} \frac{\partial^{2} T}{\partial \eta^{2}}-\left(g^{11}\left\{\begin{array}{c}
1 \\
11
\end{array}\right\}+2 g^{12}\left\{\begin{array}{c}
1 \\
12
\end{array}\right\}+g^{22}\left\{\begin{array}{c}
1 \\
22
\end{array}\right\}\right) \frac{\partial T}{\partial \xi} \\
& -\left(g^{11}\left\{\begin{array}{c}
2 \\
11
\end{array}\right\}+2 g^{12}\left\{\begin{array}{c}
2 \\
12
\end{array}\right\}+g^{22}\left\{\begin{array}{c}
2 \\
22
\end{array}\right\}\right) \frac{\partial T}{\partial \eta} \\
J=\sqrt{\left|g_{i j}\right|} & =\frac{\partial x}{\partial \xi} \frac{\partial y}{\partial \eta}-\frac{\partial y}{\partial \xi} \frac{\partial x}{\partial \eta}
\end{aligned}
$$

Sınır Şartları:

$$
\begin{array}{ll}
T(0, \eta)=0, & T(1, \eta)=0 \\
T(\xi, 0)=0, & T(\xi, 1)=100
\end{array}
$$

\section{SAYISAL YÖNTEM (NUMERICAL METHOD)}

Isı iletim denklemi ve ağ üretimi çözmek için standart sonlu farklar metodu kullanılmıştır. $\xi-\eta$ koordinatlarına göre ikinci türevlerin yaklaşımı için ikinci mertebeden merkezi farklar kullanıldı. $\xi-\eta$ koordinatlarında birincil türevler için merkezi farklar yaklaşımı kullanıldı. Bunun yanında, 1sı iletim denkleminde, $\xi-\eta$ koordinatlarında birincil türevler, birinci mertebeden upwind yoluyla ayrıklaştırıldı. Cebirsel denklemleri çözmek için Gauss-Seidel iteratif metod kullanıldı. Ağ üretim denklemleri lineer olmayan denklem sistemine sahip olduğunda, her adımda iteratif update metodu kullanılarak çözülmüştür[12]. Bu metotta denklem (8)'deki ikincil türevlerin katsayıları Gauss-Seidel itersyonunda sabit olarak ele alınmıştır ve her iterasyondan sonra güncellenmiştir. Bununla birlikte, yakınsak çözüm elde etmek için under-relaxation kullanılmalıdır.

\section{SONUÇLAR VE TARTIŞMA (RESULTS AND DISCUSSION)}

Şekil 3 örnek problem için ağ üretimini göstermektedir. Ağnın fiziksel alanın şekline uyduğu gözlemlenebilir. Sınır şartlarının uygulanması buna kolaylıkla imkân verir. Ayrıca, bu ayarlama hassasiyetin artmasina ve problemin sayısal 
çözümünün kontrolünü sağlar. Bunun ötesinde, sınırdaki ağ nokta dağılımlarının değişimi yoluyla fiziksel alanın belirli bir bölgesindeki mesh lerin konsantrasyonu mümkündür. Üretilen ağ ortogonal olmadığından, üretilen ağda esneklik sağlar.

Sayısal ağ üretim metodu oldukça çok yönlüdür ve çözüm işleminin iyi bir şekilde kontrolüne imkân verir; bununla birlikte, bu metodun da sinırlayıcıları vardır. İlk sınırlayıcı karmaşık geometriler için çok zor hatta imkânsızdır. Özellikle üç boyutlu ve çoklubağlantılı alanlarda doğrudur. Bunun yanında, hesaplama ve metrik tansörlerin ve bağlantı katsayılarının depolanması aşırı bilgisayar hafızasına ihtiyaç duymaktadır. Diğer taraftan, işlemin avantajları vardır. Birinci avantajı, sınır şartlarının uyum hassasiyeti ve hassasiyetin artmasıdır. Buna ilave olarak, bu metot yapısal bir ağ üretir ve yapısal olamayan ağlar için gerekli olan bağlantı matrisi depolanmamaktadır. Çözüm alanı hareketli ise, saysısal ă̆ üretim metodu çözüm işlemi için çok güçlü ve tutarlı bir metodoloji sağlamaktadır. Farklı zaman adımları için ağ noktaları arasında çözülen değişken için interpolasyona gerek duymamaktadır. $\mathrm{Bu}$ da hassasiyeti artırmaktadır. Bunun ötesinde, çözüm işlemi sonucunda alan değişirse, örnek olarak faz değişimi problemlerinde, sonradan şemada bu kolaylıkla yerleştirilir. Yapısal ağnın sonucunda, çözüm değişkenlerinin birinci veya ikinci mertebe türevlerine bağlı değerlerin hesaplanmasında hassasiyet kaybı olmaması önemlidir.

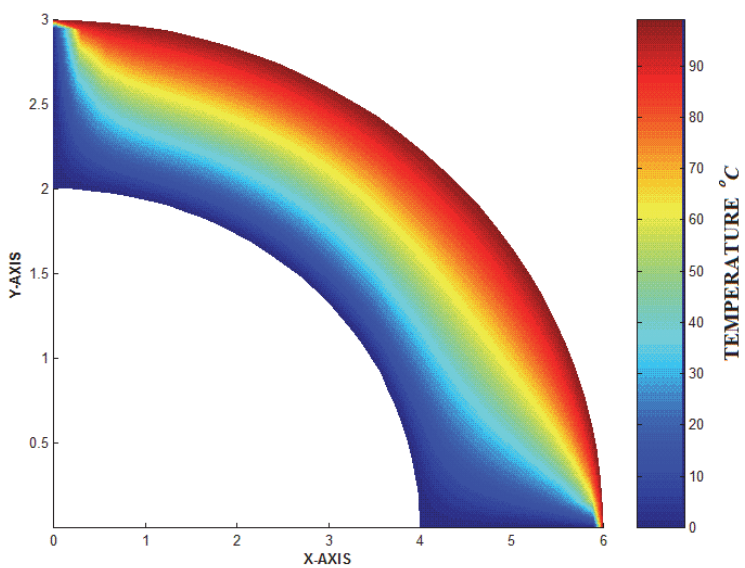

Şekil 4. Eliptik plakada kararalı durumda sıcaklık dağılımı (Steady-state temperature distribution in an elliptic plate).

Şekil 4, üretilen ağ kullanılarak tahmin edilen kararlı durumda sıcaklık konturlarını gösterir. Sınır şartlarına uygun, bir taraf $100{ }^{\circ} \mathrm{C}$ olduğu görülmekte ve diğer üç taraf $0{ }^{\circ} \mathrm{C}^{\prime}$ dir. Kararlı durumda sıcaklık dağılımı Laplace denklemi ile hesaplanmıştır. Şekil 5 Fluent den elde edilen kararlı durumdaki sicaklık konturlarını göstermektedir ve sayısal çözümü verir ve sonuçlar uyum içindedir ve Fluent kodu kullanılarak yapılan hesaplama süresi sunulan çalışmadaki sayısal çözüm süresine göre iki kat daha uzundur[13].
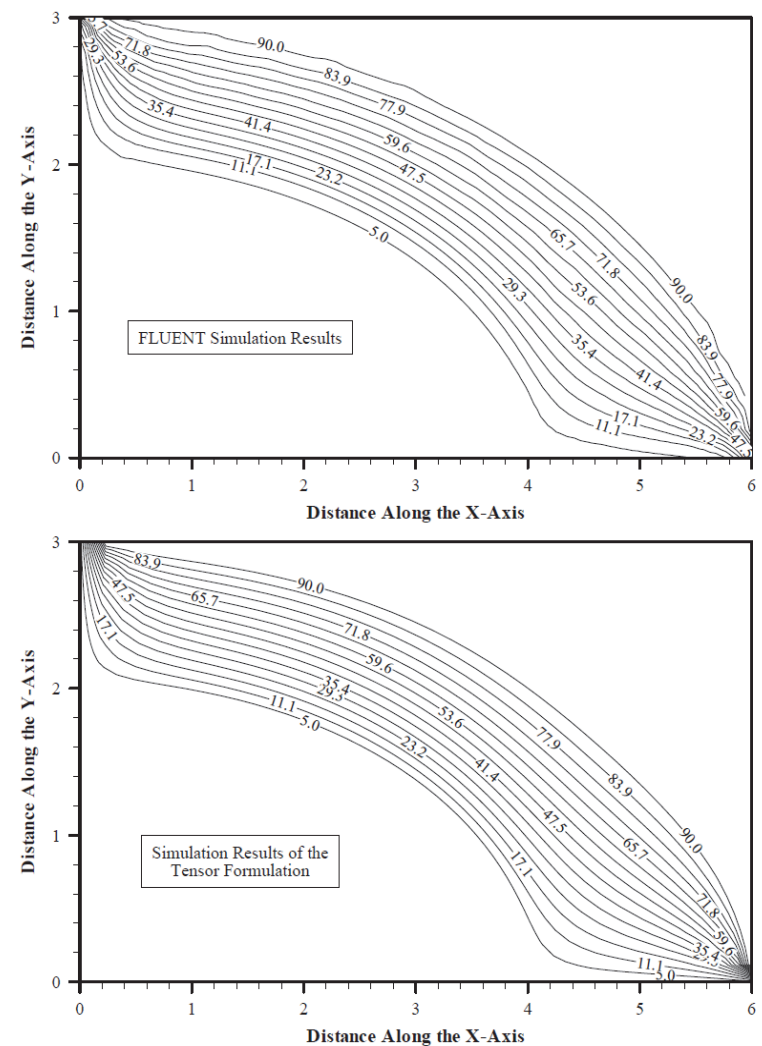

Şekil 5. FLUENT yazılımı ve geliştirilen sayısal yöntem kullanılarak elde edilen sıcaklık konturları (Temperature contours predicted using FLUENT code and present numerical method.)

\section{SONUÇ (CONCLUSION)}

Diferansiyel denklemlerin sayısal çözümü için tansör hesaplaması tanıtıldı ve tanıtılan metot geçici isı iletiferansiyel denklemlerin sayısal çözümü için tansör hesaplaması tanıtıldı ve tanıtılan metot geçici 1S1 iletdenklemine uyguland. Yönetici denklemler sayısal hesaplamalarda kolaylıkla kullanılabilen bileşen formda yazıldı. Sonuç denklemi iki boyutlu uzayı içeren herhangi bir düzlemsel koordinat sistemine uygulanabilir, üç boyutlu koordinat sistemine de kolaylıkla genişletilebilir. Düzensiz durağan düzlemsel çözüm alanları için denklemin uygulamasının mümkün olduğu ve sayısal ağ üretim metodunun geliştirilebileceği görülmektedir. $\mathrm{Bu}$ bağlamda, bu yaklaşımın faydasının gösterildiği bir örnek sunulmuştur. Isı iletim denklemlerinin (5 \& 7) formu Reimannian (non-Euclidean) uzayına uygulanabilir, bununla birlikte küresel kabuğun iki boyutlu yüzey olarak dönüştürüldüğüne dikkat edilmelidir.

\section{TEŞEKKÜR (ACKNOWLEDGEMENTS)}

King Fahd University of Petroleum \& Minerals'a çalışmamıza sağladığı destek için teşekkür ederiz. 


\section{KAYNAKLAR (REFERENCES)}

1. Thompson, J.F., Warsi, Z.U.A., Mastin, C.W., "Numerical Grid Generation, Foundations and Applications", Elsevier Science Publishing Co., Inc., 1985.

2. Wathen, A.J., "Time-resolved observation of electron-phonon relaxation in copper," Phys Rev Lett, Cilt 58, 1212-1215, 1992.

3. McCorquodale, P., Colella, P., Johansen, H., "A Cartesian grid embedded boundary method for the heat equation on irregular domains," Journal of Computational Physics, Cilt 173, No 2, 620-635, 2001.

4. Beckett, G., Mackenzie, J.A., Robertson, M.L., "A moving mesh finite element method for the solution of two-dimensional Stefan problems," Journal of Computational Physics, Cilt 168, No 2, 500-518, 2001.

5. Kuvyrkin, G.N., Titov, A.V., "On problems of heat conductivity with a moving boundary,"
Izvestiya Vysshikh Uchebnykh Zavedenii, Mashinostroenie, Cilt 12, 136-139, 1979.

6. Gilmore, S.D., Guceri, S.I., "Three-dimensional solidification, a numerical approach", Numerical Heat Transfer, Cilt 14, No 2, 165-186, 1988.

7. Chin, J.H., "Finite element analysis for conduction and ablation moving boundary," AIAA Pap AIAA Thermophysics Conference, $15^{\text {th }}$, July 14-16, 1980.

8. Aris, R., "Vectors, tensors, and the basic equations of fluid mechanics", Dover Publications, 1990.

9. Synge, J.L., "Tensor Calculus". University of Toronto Press, 1969

10. Heinbockel, J.H., "Introduction to Tensor Calculus and Continuum Mechanics". Trafford Publishing, 2001.

11. Truesdell, C., "The Physical components of vectors and Tensors," Journal of Applied Math. Mech., Cilt 33, 245-356,1953.

12. Ozisik, M.N., "Finite Difference Methods in Heat Transfer". CRC Press, 1994.

13. Fluent Users Guide, Fluent Inc. 2010. 
